\title{
CHARACTERIZATION OF THE VERY LOW CONTACT RESISTANCE ON HEAVILY BORON DOPED (113) CVD DIAMOND
}

\author{
1'Jan VOVES, ${ }^{1}$ Alexandr LAPOSA, ${ }^{2}$ Zbyněk ŠOBÁŇ, ${ }^{1}$ Pavel HAZDRA, ${ }^{1}$ Vojtěch POVOLNÝ, \\ ${ }^{2,3}$ Vincent MORTET, ${ }^{1,2}$ Nicolas LAMBERT, ${ }^{2}$ Marina DAVYDOVA, \\ ${ }^{1}$ Faculty of Electrical Engineering, Czech Technical University in Prague, Prague, Czech Republic, EU, \\ voves@fel.cvut.cz \\ 2Institute of Physics of the Czech Academy of Sciences, Prague, Czech Republic, EU, mortet@fzu.cz \\ ${ }^{3}$ Faculty of Biomedical Engineering, Czech Technical University in Prague, Kladno, Czech Republic, EU
}

https://doi.org/10.37904/nanocon.2021.4320

\begin{abstract}
The low resistance of ohmic contacts on diamond layers is important for the fabrication of diamond power electronic devices with fast switching capabilities for future high voltage applications. The low barrier height between the metal and diamond, high level of boron doping and annealing at elevated temperatures are the most critical parameters to reach the lowest contact resistivity. In this work, we report on titanium/gold ohmic contacts prepared on the heavily boron-doped (113) epitaxial diamond layers. The contact resistance has been characterized by the Circular Transmission Line Model (cTLM) structures. We used the analytical model of field enhanced emission, tunneling and the image force influence including Fermi level position dependence on the boron concentration for theoretical Ti/Au contact analysis and the Silvaco TCAD 2D simulation to estimate the measurement error associated with the nonzero metal resistance. We show that the resulting simulation values are consistent with the experimental results.
\end{abstract}

Keywords: Diamond, ohmic contacts, power devices, TCAD simulation

\section{INTRODUCTION}

CVD diamond layers with (113) surface orientation are studied as a new platform for preparation of high-performance diamond power devices [1,2]. They offer better surface morphology and lower surface roughness in comparison with (100) and (111) oriented substrates. High-power diamond devices require reliable and low-resistance ohmic contacts to minimize their total $\mathrm{ON}$-state resistance [3]. In this work, we compare the experimental electrical properties of Ti/Au ohmic contacts on the epitaxial (113) highly borondoped diamond (BDD) with theoretical calculations based on the CTLM analytical method and on the physical TCAD simulations. This analysis is necessary for the accurate determination of very low values of contact resistance on highly conductive diamond layers, the sheet resistance of which is comparable to the resistance of metallization.

\section{EXPERIMENTAL}

BDD layers were grown on (113) oriented substrates in a commercial microwave plasma enhanced chemical vapor deposition (MWPECVD) reactor AX5010 from Seki Diamond Systems. The microwave power was 700 $\mathrm{W}$, pressure 100 mbar, methane concentration $1 \%$, and different $\mathrm{B} / \mathrm{C}$ ratios in the gas phase (100 to 2000 ppm) provided boron concentrations in the epilayer between $10^{19}$ to $10^{21} \mathrm{~cm}^{-3}$. The surface morphology and the thickness of deposited layers were determined by atomic force (AFM) and cross-section scanning electron microscopy (X-SEM). Their resistivity, carrier concentration, and carrier mobility at room temperature were determined by Hall effect measurement. To characterize contact properties, circular Transmission Line Model 
(cTLM) patterns using a $75 \mu \mathrm{m}$ radius of the inner circle $(L)$ and 10 to $75 \mu \mathrm{m}$ electrode gap spacing $(d)$ to the outer electrode were prepared using a laser lithography system (Microwriter $\mathrm{ML}$ ) and wet chemical etching (shown in Figure 1). The $10 \mathrm{~nm}$ thick Ti contact layers covered by a $100 \mathrm{~nm}$ Au capping layer were evaporated using a Covap PVD system from Angstrom Engineering with a base pressure below $3 \times 10^{-7}$ mbar on an ozone treated (113) surface and annealed at temperature up to $700^{\circ} \mathrm{C}$. Titanium (Ti) is one of the most commonly used metals to fabricate ohmic contacts on boron-doped diamond. Ti ohmic contacts covered by the gold capping layer have a good thermal stability. The Kelvin method, Cascade Microtech M150 probing system and an Agilent B4156A semiconductor parameter analyser were used for the measurement of current-voltage (IV) characteristics. Contact resistances $R_{C s p}$, transfer lengths $L_{T}$ and sheet resistances $R_{s h}$ were obtained by the linear least square fitting of the dependence of the cTLM structure resistance $R_{T}$ with $d$, which is valid only when the $d$ is much smaller than $L[4]$.
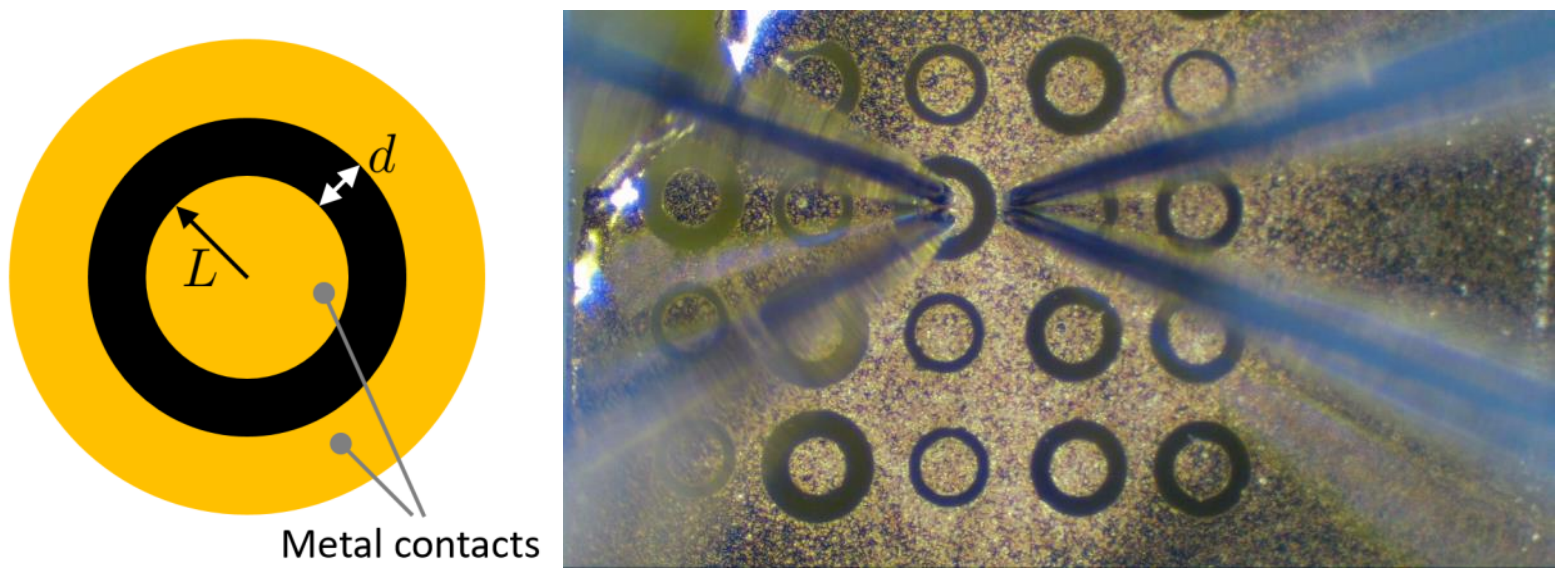

Figure 1 The cTLM structure used for measurement of the contact resistance on the BDD layers. The schematic picture (left) and the photo of four-probe measurement of the real structure (right)

\subsection{The correction factor}

In the case when $d \geq 4 L$ the measured resistance should be multiplied by the correction factor $C$ during the $R_{c}$ determination. The total measured C-TLM resistance is given by [4]:

$R_{T}=\frac{R_{S h}}{2 \pi L}\left(d+2 L_{T}\right) C$

where $R_{s h}$ is the diamond sheet resistance, $L_{T}$ is the transfer length where the current flows from metal to semiconductor layer and the correction factor is defined by:

$C=\frac{L}{d} \ln \left(1+\frac{d}{L}\right)$

The corrected resistance value $R_{\mathrm{Tcorr}}$ is then linearly dependent on $d$ according to:

$R_{T_{\text {corr }}}=\frac{R_{T}}{C}=\frac{R_{S h}}{2 \pi L}\left(d+2 L_{T}\right)$

As shown in Figure 2, where the open squares are the measured values and full squares are the corrected values, we can see the perfect linear behaviour in respect to the gap spacing $d$. The gradient multiplied by $2 \pi L$ gives the sheet resistance of the BDD layer and the specific contact resistance is calculated as:

$R_{C_{S P}}=R_{S h} 2 L_{T}^{2}$ 


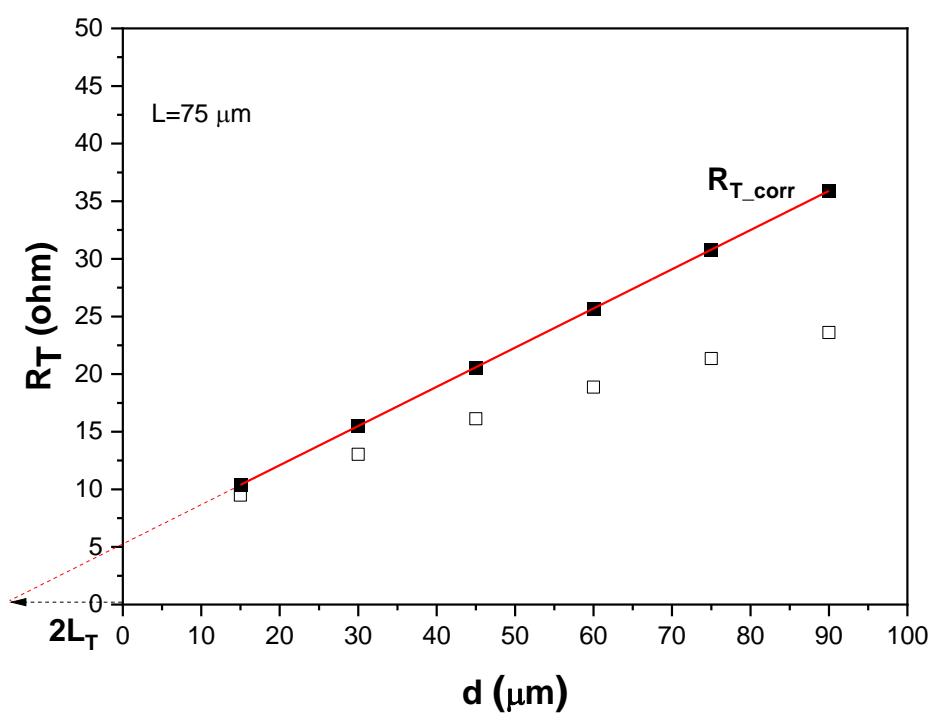

Figure 2 The total resistance of the cTLM structure in the dependence on the gap spacing. Experimental results are the open squares and corrected values are the full squares with the linear fit.

\section{MODELS OF THE CONTACT RESISTANCE}

\subsection{The analytical model}

To determine the barrier height $\Phi_{b}$ between the Ti/Au and p-type diamond we used the analytical model of the contact resistance of the Schottky diode [5 - 7]. The built-in voltage $V_{b i}$ is defined by:

$V_{b i}=\Phi_{b}-\frac{1}{q}\left(E_{F}-E_{V}\right)$

Where $\Phi_{b}$ is the barrier height and $E_{F}-E_{V}$ is the distance between Fermi level and the valence band energy:

$E_{F}-E_{V}=k_{B} \mathrm{~T} \cdot \ln \left(\frac{N_{V}}{N}\right)$

$N_{V}$ is the valence band effective density of states equal to $10^{19} \mathrm{~cm}^{-3}$ for diamond at room temperature [8] $T$ is the absolute temperature, $k_{B}$ is the Boltzmann constant and $N$ is the doping concentration. We included the Schottky barrier lowering $\Delta \varphi_{b}$ due to image force:

$\Phi^{\prime}{ }_{b}=\Phi_{b}-\Delta \Phi_{b}=\Phi_{b}-\left(\frac{q^{3} N\left(V_{b i}-\frac{k_{B} T}{q}\right)}{8 \pi^{2}\left(\varepsilon_{r} \varepsilon_{O}\right)^{3}}\right)^{\frac{1}{4}}$

where $\varepsilon_{r}$ is the diamond relative permittivity (5.5) in [9]. For the thermionic field emission model of the charge transfer the contact resistance $\rho_{C(T F E)}$ is given by:

$\rho_{C(T F E)}=c_{T F E} \frac{k_{B}}{q A^{*} T} \exp \left(\frac{q \Phi_{b}^{\prime}}{E_{0}}\right)$

where $A^{*}$ is the modified Richardson constant [10] and the coefficient $c_{T F E}$ is calculated by the formula:

$c_{T F E}=\frac{k_{B} T \cosh \left(\frac{E_{00}}{k_{B} T}\right) \sqrt{\operatorname{coth}\left(\frac{E_{00}}{k_{B} T}\right)}}{\sqrt{\pi\left(q \Phi^{\prime}{ }_{b}+\left(E_{F}-E_{V}\right)\right) E_{00}}} \exp \left(\frac{E_{F}-E_{V}}{E_{0}}-\frac{E_{F}-E_{V}}{k_{B} T}\right)$

where the exponential term can be approximated by 1 for higher doping level and 
$E_{0}=E_{00} \cdot \operatorname{coth}\left(\frac{E_{00}}{k_{B} T}\right)$

with

$E_{00}=\frac{q \hbar}{2} \sqrt{\frac{N}{m_{t}^{*} \varepsilon_{r} \varepsilon_{0}}}$

$m_{t}^{*}$ is hole effective mass $\left(0.908 m_{0}\right.$ for diamond) [8]. At high diamond doping levels, the field emission (tunnelling) is the most important transport mechanism. The contact resistance is given by:

$\rho_{C(F E)}=c_{F E} \frac{k_{B}}{q A^{*} T} \exp \left(\frac{q \Phi_{b}^{\prime}}{E_{00}}\right)$

where

$c_{F E}=\frac{q}{E_{00}} \quad$.

The resulting contact resistance is determined from the formula for parallel combination of both:

$\rho_{C(F E, T F E)}=\left(\frac{1}{\rho_{C(F E)}}+\frac{1}{\rho_{C(T F E)}}\right)^{-1}$

\subsection{The TCAD model}

The influence of the finite resistivity of the Ti/Au layer was inspected by the Silvaco TCAD two dimensional simulation. In the ideal case, we expect that the isotropic current flows between the outer and inner electrode during cTLM measurement. In the real case, the finite resistivity of the metal layer creates nonisotropic potential distribution at the circular structure. For the simulation, we have chosen the silicon model with the concentration $10^{19} \mathrm{~cm}^{-3}$, thickness $1 \mu \mathrm{m}$ and the artificial mobility of $18199 \mathrm{~cm}^{2} / \mathrm{V}$.s which gives the same resistivity as our Ti/Au layer (the real case). For the simulation of an „ideal contact metal“, we increased the carrier mobility of $\mathrm{Ti} / \mathrm{Au}$ layer 100 times. The difference in resistance between the real and ideal case is larger with the lower ratio between the sheet resistance of the diamond and the metal layers as shown in the Figure 3 for the $L=75 \mu \mathrm{m}$ and $d=20$ and $75 \mu \mathrm{m}$ with the probe distance $200 \mu \mathrm{m}$. The simulated distributions of current densities in the C-TLM structure corresponding to the real case are shown in Figure 4.

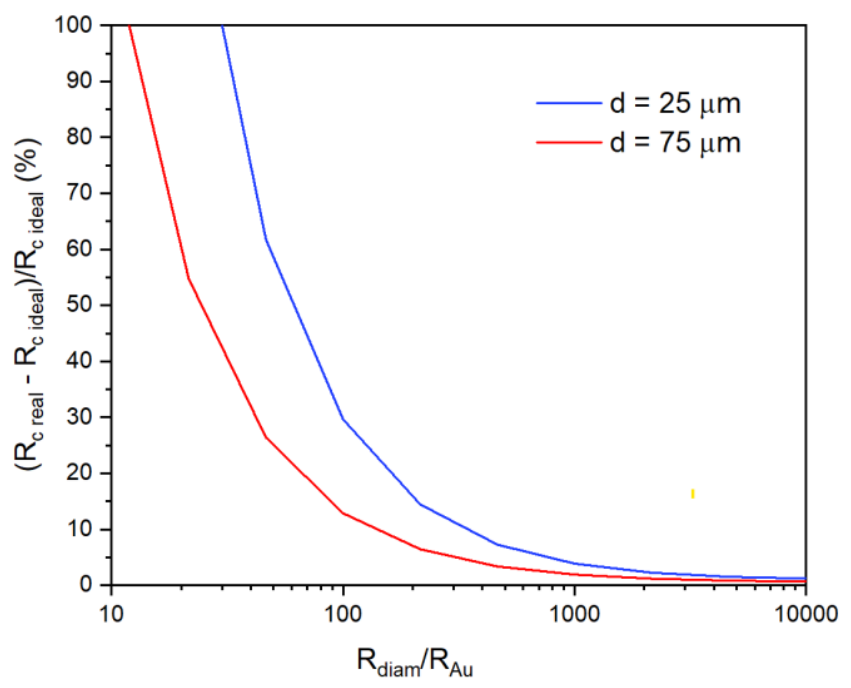

Figure 3 The relative difference between the ideal and real cTLM structure in the dependence on the diamond/metal sheet resistance ratio. 


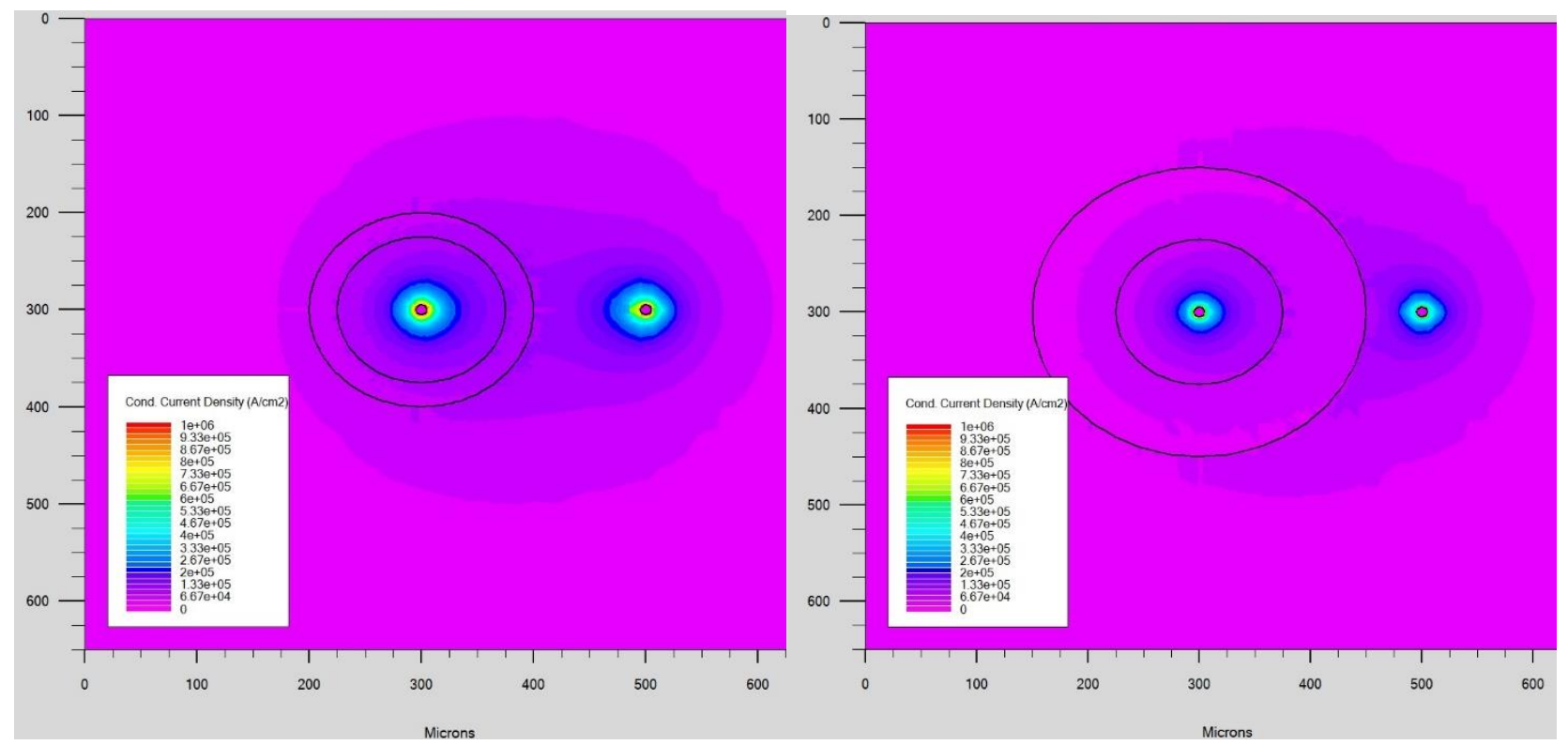

Figure 4 The simulated two-dimensional plot of the current density with $d=20$ and $75 \mu \mathrm{m}$ and the sheet resistance of the metal layer only 10 times lower than for the diamond layer.

\section{RESULTS}

We compared the result of analytical model with the experimental values of cTLM method. The result is shown in the Figure 5.

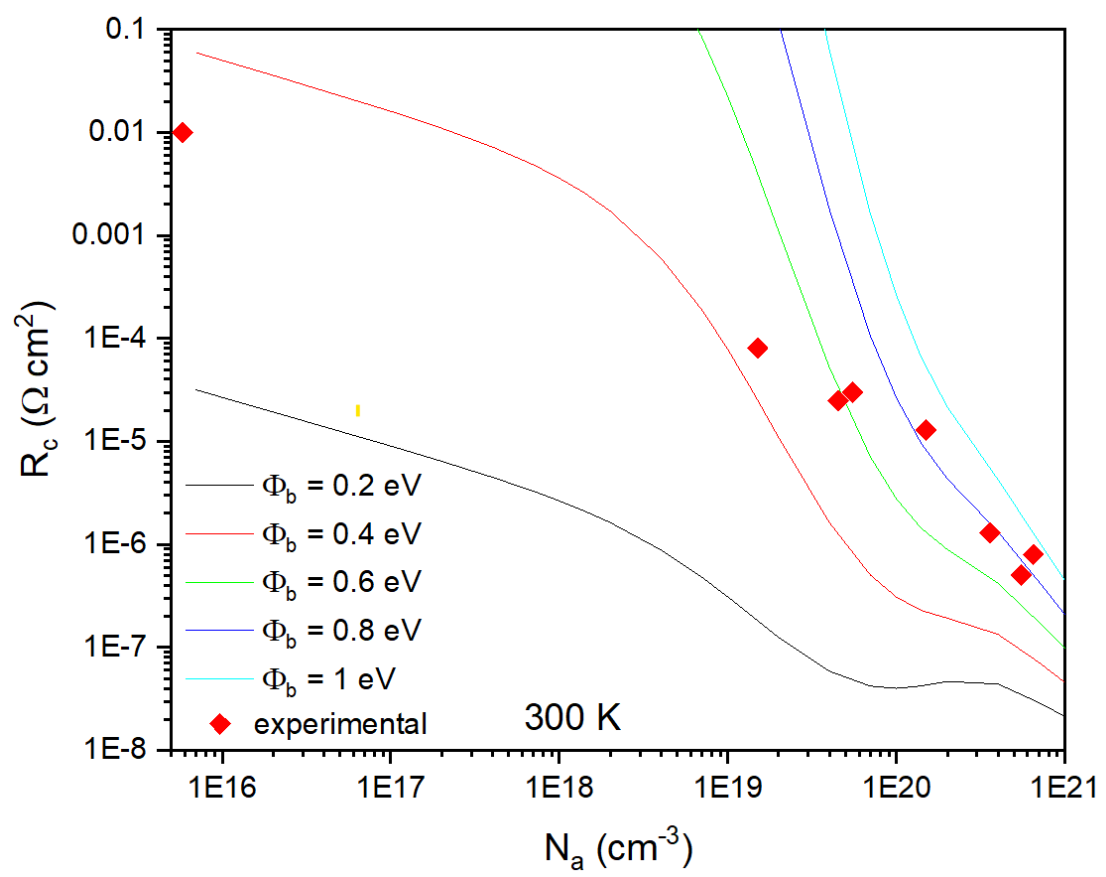

Figure 5 The calculated contact resistances in the dependence on the diamond boron doping levels for different contact barrier heights together with the experimental results.

The lowest values of the specific contact resistance reached was around $10^{-6} \Omega . \mathrm{cm}^{2}$, which is the resolution limit of the CTLM method in our configuration. At lower diamond boron doping levels the experimental data are close to the curve with barrier height $0.4 \mathrm{eV}$, which is consistent with the published values [11]. We found out 
a significant decrease of the contact resistance compared to the values, which we obtained on epilayers with (100) and (111) orientation [3]. Moreover, Ti/Au contacts showed a stable ohmic behaviour in the whole range of annealing temperatures.

\section{CONCLUSION}

This study confirms the high suitability of (113) oriented diamond epitaxial layers in the combination with Ti/Au metal layers for the fabrication of diamond power electronic devices with low resistance ohmic contacts. The cTLM method could be used for the specific contact resistance characterization. The correction of the results due to low $L / d$ ratio are important to include and the error connected with the low diamond/metal sheet resistance ratio should be taken into account.

\section{ACKNOWLEDGEMENTS}

This work was supported by the Czech Science Foundation grant No. 20-11140S by the Czech Technical University Student Grant No. SGS20/176/OHK3/3T/13 and by the Ministry of Education, Youth and Sports of the Czech Republic project LM2018110.

\section{REFERENCES}

[1] PINAULD-THAURY, M.-A. et al. Phosphorus-doped (113) CVD diamond: A breakthrough towards bipolar diamond devices. Appl. Phys. Lett. 2019, vol. 114, p. 112106.

[2] MORTET, M. et al. Properties of boron-doped (113) oriented homoepitaxial diamond layers, Diam. Relat. Mater. 2021, vol. 111, p. 108223.

[3] KOIZUMI, S. et al. Power Electronics Device Application of Diamond Semiconuctors. Woodhead Publishing, 2018.

[4] KLOOTWIJK, J.H., TIMMERING, J.H. Merits and Limitations of Circular TLM structures for contact resistance determination for novel 111-V HBTs, In Proc. of IEEE 2004 Int. Conference on Microelectronic Test Structures, Avaji, IEEE. 2004, pp. 247-252.

[5] LOHMÜLLER, E., WERNER, S., HOENIG, GREULICH, R.- J., CLEMENT, F. Impact of boron doping profiles on the specific contact resistance of screen printed Ag-Al contacts on silicon, Solar Energy Materials \& Solar Cells 2015, vol. 142, pp. 2-11.

[6] VARAHRAMYAN, K., VERRET, E. J., A Model for Specific Contact Resistance Applicable for Titanium SilicideSilicon Contacts. Solid-Slate Electronics. 1996, vol. 39, pp. 1601-1607.

[7] YU, A. Y. C. Electron Tunneling and Contact Resistance Metal-Silicon Contact Barriers. Solid-State Electronics. 1970, vol. 13, pp. 239-247.

[8] FONTAINE, F. Holes in boron-doped diamond: comparison between experiment and an improved model, Diamond and Related Materials. 2000, vol. 9, pp. 1076-1080.

[9] DONATO, N. et al., Diamond power devices: state of the art, modelling, figures of merit and future perspective, J. Phys. D: Appl. Phys. 2020, vol. 53, p. 093001.

[10] CROWELL, C. R. The Richardson Constant for Thermionic Emission in Schottky Barrier Diodes, Solid-State Electronics. 1965, vol. 8, pp. 395-399.

[11] LI, F. N. Barrier heights of Au, Pt, Pd, Ir, Cu on nitrogen terminated (1 000 ) diamond determined by X-ray photoelectron spectroscopy. Appl. Surf. Sci. 2018, vol. 456, pp. 532-537. 